\title{
An independent evaluation of the national programme for the elimination of lymphatic filariasis
}

\author{
Yahathugoda TC, Weerasooriya MV, Samarawickrema WA \\ Filariasis Research Training and Service Unit, Department of Parasitology, Faculty of Medicine, University of \\ Ruhuna, Galle, Sri Lanka
}

Correspondence: Dr.T.C.Yahathugoda (tcyahath@yahoo.co.uk)

\begin{abstract}
This review covers studies carried out by an independent research group related to the national programme for the elimination of lymphatic filariasis. Research carried out under each step of the elimination ladder provided new information to assist future activities of the programme. For disease mapping a Rapid assessment procedure (RAP) has been validated. A positive significant correlation was established between ordinary peoples' information on filariasis prevalence and actual infection status based on clinical and immunological examinations. The data on hydrocoele, elephantiasis and socioeconomic indicators obtained by RAP through GNs of Hambantota, Matara and Galle were subjected to Geographical Information System (GIS) using TNTmips software and maps were prepared to analyse the disease distribution.

National Mass Drug Administrations (MDAs) were monitored and evaluated. Overall drug coverage had reached $80 \%$ except in municipality areas. The community-wide treatments suppressed the $\mathrm{mf}$ prevalence and density to significantly low levels. The effect of MDAs on soil-transmitted helminthiasis was also studied. A significant decline was observed in the prevalence. At verification stage, sites in endemic and non-endemic zones were checked with most reliable urine ELISA method. Results showed low level of ongoing transmission at non-threatening level.

Before lymphoedema management programme, the information on lymphoedema and its management was collected. Many had acute inflammatory episodes (AIEs) with fever. Components of lymphoedema management protocol (LMP) were ignored by many. LMP was then applied to a sample of 27 patients: 14 were monitored daily as daily follow-up group (DFU) and 13 once a month as monthly follow-up group (MFU). Evaluations were carried out at one year and found that the benefits received were significantly higher in DFU.
\end{abstract}

\section{Introduction}

The national Anti-Filariasis Campaign (AFC) was inaugurated in 1947 when Wuchereria bancrofti infection was confined to coastal endemic belt extending from Negombo to Matara and several high endemic foci of Brugia malayi were located at Southern, Western Northwestern and Eastern provinces (Figure 1). Later B. malayi prevalence was dramatically reduced and isolated to a few pockets Induruwa and Boosa. By 1967 B. malayi infection completely disappeared from the country (1). An expanding endemic belt of $W$. bancrofti was recorded by $\operatorname{AFC}(2)$.
The national programme and concurrent programme conducted by the Filariasis Research Unit, Faculty of Medicine, University of Ruhuna, Galle (FRTSU)

Following the Global Programme to Eliminate LF (GPELF) the Ministry of Health initiated the national programme (national PELF) in 2002 (Figure 2) (5). The national task force appointed director anti-filariasis campaign as the programme manager. The goal for achieving elimination was 2020. The two principal strategies were interruption of transmission and disability prevention and control. 
Our team, Filariasis Research Unit (FRTSU), University of Ruhuna, formed the main research arm of the national PELF and an independent group to monitor and evaluate its activities.

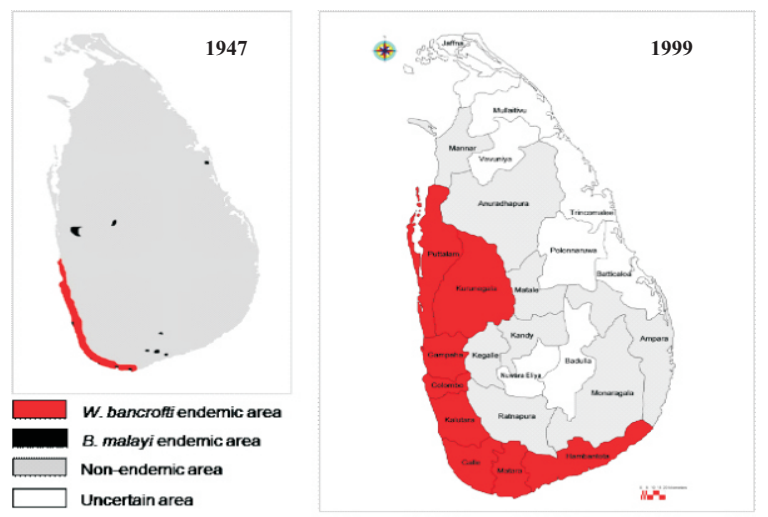

Figure 1: Distribution of endemic areas of $W$. bancrofti and $B$. malayi as observed in the microfilaria surveys conducted in 1947 (3) and $1999(4)$

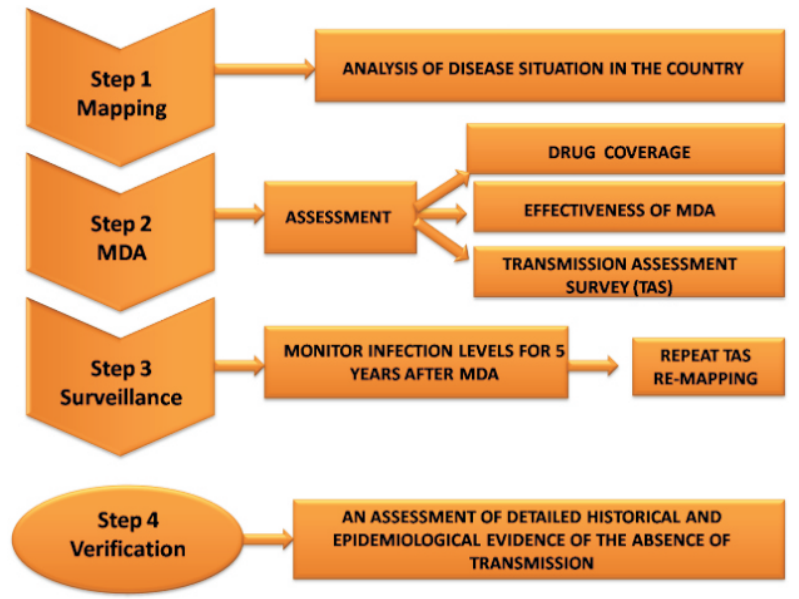

Figure 2: Programmatic steps taken by the national PELF

Step 1 - Analysis of disease situation in the country - mapping

In 1999, AFC mapped out bancroftian filariasis distribution into eight districts, three in the Southern province, three in Western province and two in North-Western province (Figure 1).

Initially FRTSU screened the disease status of the three districts in Southern Province. Rapid Assessment Procedure (RAP) was used with modification to suit the local situation (6). Validation of RAP as a useful tool in the assessment of LF endemicity in Lankan context was carried out. In Matara district we found several inland villages with hydrocoele cases (7). Transmission in these inland areas was confirmed by immunodiagnosis $(\mathrm{Og} 4 \mathrm{C} 3$ ELISA). Fifteen out of 2436 school children were positive for $W$. bancrofti antigen. Indirect questionnaire method (IndQ) (RAP) was validated using hydrocoele prevalence ( $\%$ males) by clinicians (CE) and urine ELISA (IgG4) in a study carried out at Hambantota (8). Results of above study showed significant correlation between CE and urine ELISA (Pearson's correlation analysis $r=0.767, P<0.001$ ). To examine to what extent the two RAPs by people's information can predict professionals' results on hydrocoele and filarial antibody positivity, the logistic regression analysis was conducted with $\mathrm{CE}$ and urine ELISA rates as the dependent variables. Each of IndQ and Cluster-IndQ rate was set as a single predictor. The predictiveness of these RAP variables were evaluated with odds ratio (OR) per one case (/1,000 male population) increase of hydrocoele by IndQ and Cluster-IndQ. In addition, the goodness of the fit was compared by pseudo- $\mathrm{R}^{2}$ defined as: $\left(l_{\text {full }}-l_{\text {model }}\right) /\left(l_{\text {full }}-l_{\text {mull }}\right)$, where $l_{\text {full }} l_{\text {model }}$, and $l_{\text {null }}$ are, respectively, log-likelihood of the full model with $G N$ code as categorical independent variable, that of the model tested, and that of the null model with intercept only (Figure $3 \& 4$ ).

\section{3a [CEvs. IndQ]}

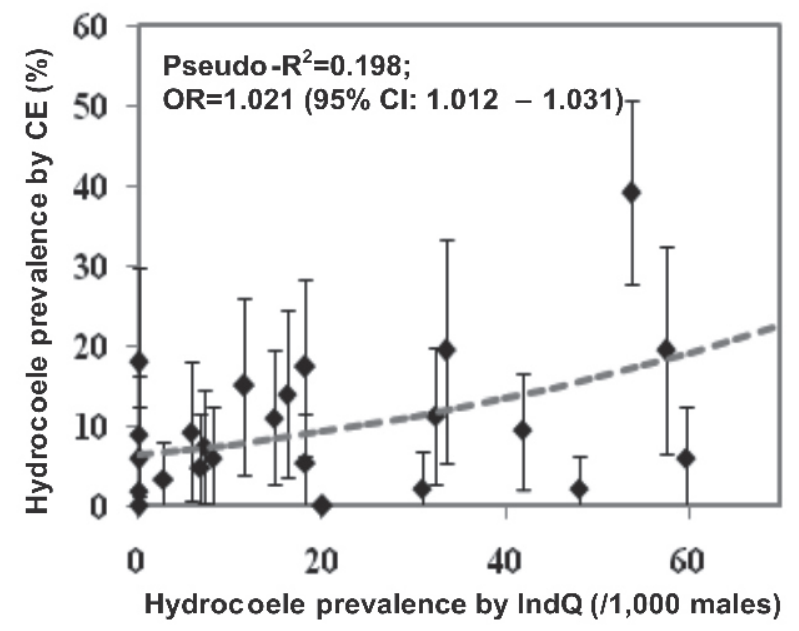

Figure 3: Relationship between hydrocoele prevalence by $\mathrm{CE}$ and that by IndQ, or ClusterIndQ in 24 GN divisions 


\section{3b [CE vs. Cluster-IndQ]}

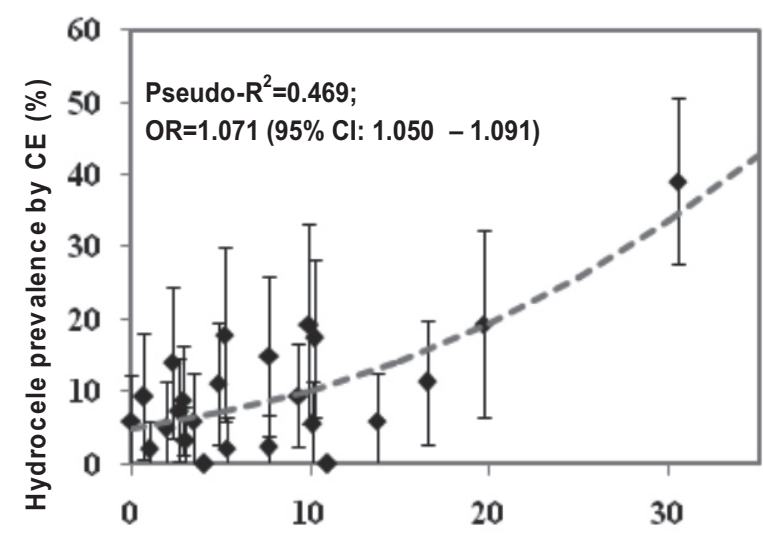

Hydroc oele prevalence by Cluster-IndQ(/1,000 males)

\section{4a [Urine ELISA vs. IndQ]}

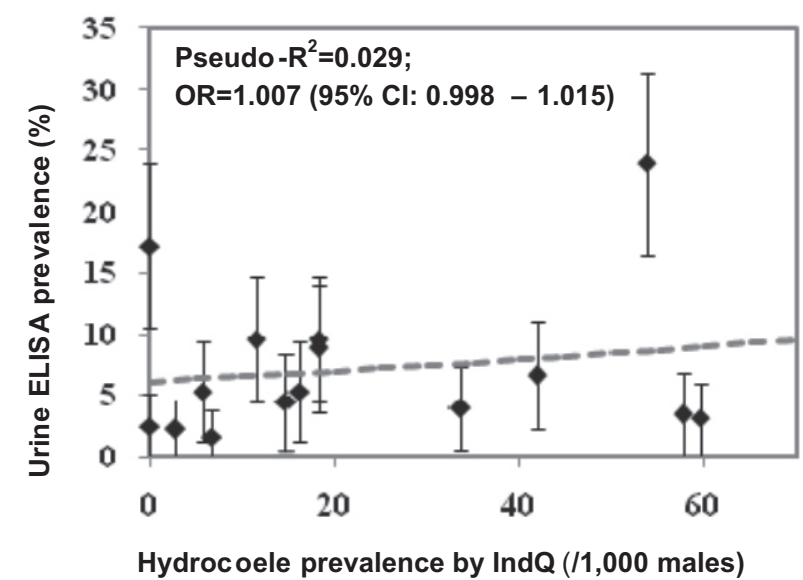

4b [Urine ELISA vs. Cluster-IndQ]

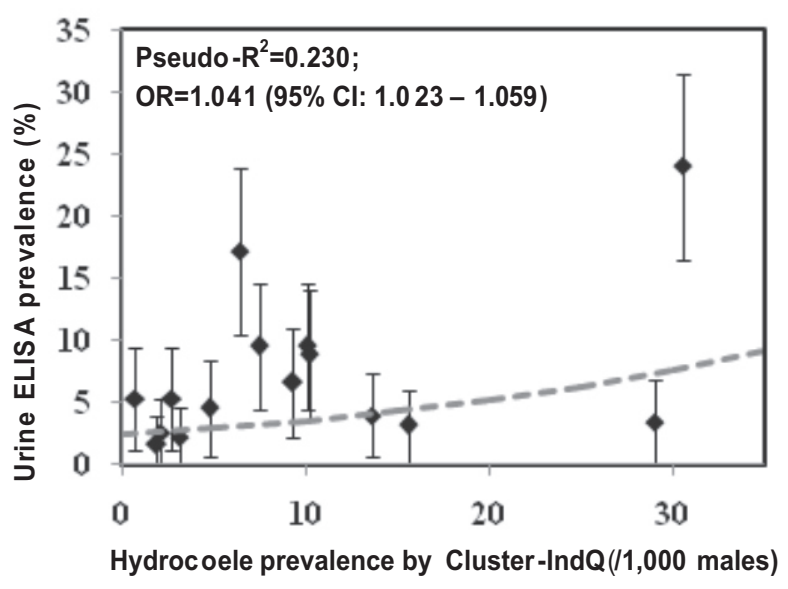

Figure 4: Relationship between urine ELISA prevalence and hydrocoele prevalence by IndQ or Cluster-IndQ in 15GN divisions
Our data clearly showed that questionnaire (IndQ) can be used for rapid assessment for hydrocoele prevalence, especially when it is averaged (smoothed) with the neighbor areas. The relationship was less clear between IndQ and seroprevalence of filariasis, possibly because low number of Grama Niladhari $(G N)$ divisions $(\mathrm{n}=15)$ were tested with urine ELISA compared to CE $(\mathrm{n}=24)$. Nevertheless, the averaged (smoothed) data showed significant positive relationship with urine ELISA.

A similar RAP study was also carried out covering Galle district. All IndQ data of Hambantota, Matara and Galle were expressed in GIS maps (9). RAP covering Hambantota, Matara and Galle showed a comparable disease distribution. The elephantiasis cases were confined to coastal villages while hydrocoele cases were heterogeneously distributed (Figure 5). These findings justified the selection of the whole district as an implementation unit by the PELF.

\section{Step 2 - Interruption of Transmission by Mass Drug Administration (MDA)}

Areas with $>1 \%$ microfilaraemia were considered for MDA (10). However routine surveys by AFC during 1981 to 1998 showed very low mf rates of 0.23 to 0.38 (4). Our surveys in Matara showed relatively high $\mathrm{mf}$ rates (Polhena $=3.3$; Madihe $=5.6$; Walgama $=5.7)(11)$. In the light of our data the national PELF decided to cover all three provinces, southern, western and north western with MDA (Figure 1). The national MDA commenced in 2002 and continued annually through to 2006. Drug delivery to the population was mainly by two methods, house to house delivery by volunteers and through delivery centres. The treatment programme consisted of DEC $(6 \mathrm{mg} / \mathrm{kg})$ and Albendazole (400mg).

\section{Drug coverage assessments}

FRTSU conducted a series of evaluation studies. (1) In 2002 we compared the awareness of the MDA in two communities, urban and rural, in the Galle district (12). Awareness of the people on the MDA increased from $40.2 \%$ to $99.6 \%$. However, drugs coverage was $76.9 \%$ in Unawatuna (urban) compared with $89.0 \%$ in Baddegama (rural) population. (2) Next in 2003 we visited all eight districts covering a sample of 4358 subjects (13). 
The coverage in urban and municipality areas was poor. House to house coverage was far more successful than coverage from delivery centres. (3) To confirm low coverage in the municipality areas we evaluated the $2004 \mathrm{MDA}$ in the 47 wards of the Colombo municipality and 12 wards in Matara municipality (14). We classified the Colombo wards into (A) mostly commercial (B) upper class residential (C) mostly middle class residential (D) mostly thickly populated slum housing. Awareness coverage was lowest in the mostly commercial and upper class housing wards. Two of the upper class housing wards recorded below 20\%. Matara municipality showed much improved Figures of 97.8\% awareness of the MDA and $78.1 \%$ drug coverage compared to Colombo. (4) The Galle municipality has 15 wards. We classified them into (a) commercial and upper class housing (b) middle, working class and slum housing. We first evaluated the 2003 MDA programme in the municipality.
Three teams from FRTSU carried out a model programme, one team for IEC, another for drug coverage and the third for evaluation (15). A team covered the entire municipality in one day. Two delivery centres, Health office and Faculty of Medicine operated for two further weeks. Finally, a mobile distribution centre was used in mop-up coverage. An independent third team carried out an evaluation of the coverage. Improvement in awareness was significant in both housing categories $(75 \%$ to $93 \%-P<0.0001)$ and coverage (a - 57\% to $79 \%$ : b $-75 \%$ to $90 \%, P<0.0001$ ). (5) On our recommendations national PELF had improved vastly after 2004 MDA. National programme was evaluated in the districts of Hambantota, Matara and Galle in 2005 and 2006. Fifteen villages were selected from three strata - coastal, intermediate and inland - from each district. $100 \%$ geographical coverage and $>80 \%$ drug coverage were observed (Reports were submitted to AFC and Ministry of Health).
Prevalence (/1000)

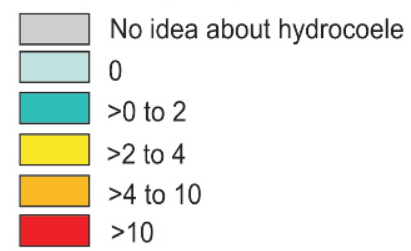

(c)

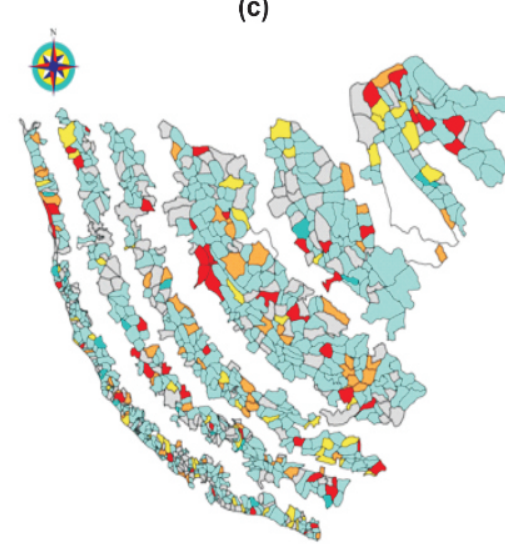

(b)

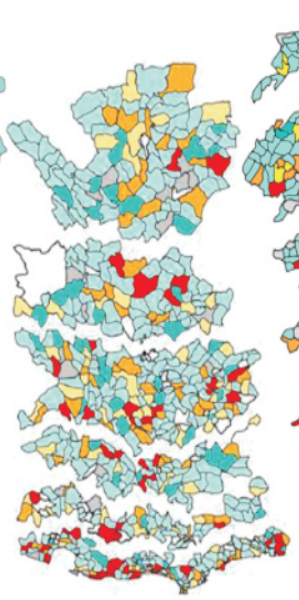

(a)

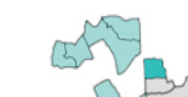

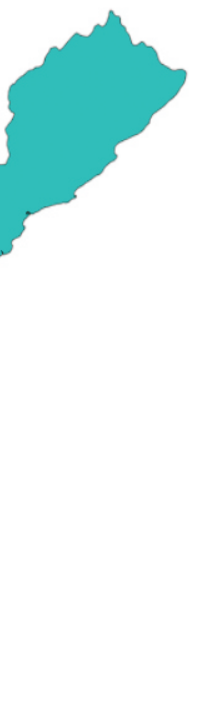

Figure 5: Hambantota (a), Matara (b), and Galle (c), prevalence maps of hydrocoele according to distance from the coastline 


\section{Effectiveness of MDA and Transmission Assessment Survey (TAS)}

We conducted a model programme from 2001 to 2008 covering Walgama, Matara where the preMDA mf rates were relatively high (16). Our coverage in 4 treatment areas Hamugewatta, Matotagma [sub-division], Walgama and Walgama Central through out exceded $96 \%$.

Investigations included assessing microfilaria rates, vector rates and helminth rates. Prior to each MDA we carried out mf surveys and stools surveys in selected target populations. $60 \mu \mathrm{L}$ finger pricked blood samples taken from $9.00 \mathrm{pm}$ to $12.00 \mathrm{pm}$ were examined for microfilaria. Faecal samples were screened using Kato-Katz. Vector infections were identified from dissection of samples from monthly vector catches (17).

Walgama had the lowest $(3.7 \%)$ pre-MDA $\mathrm{mf}$ prevalence and recorded less than $0.5 \%$ prevalence after six rounds of annual MDAs (Figure 6). Hamugewatta (8.6\%) and Matotagama [sd] (5.7\%), whose pre-MDA $\mathrm{mf}$ rates were relatively high, also showed a significant reduction following 12 rounds of biannual MDAs (18). However, Hamugewatta recorded $>1 \% \mathrm{mf}$ rate even after 12 rounds of MDA where another round of MDA has been conducted. Manuscript covering above mentioned work yet to be published.

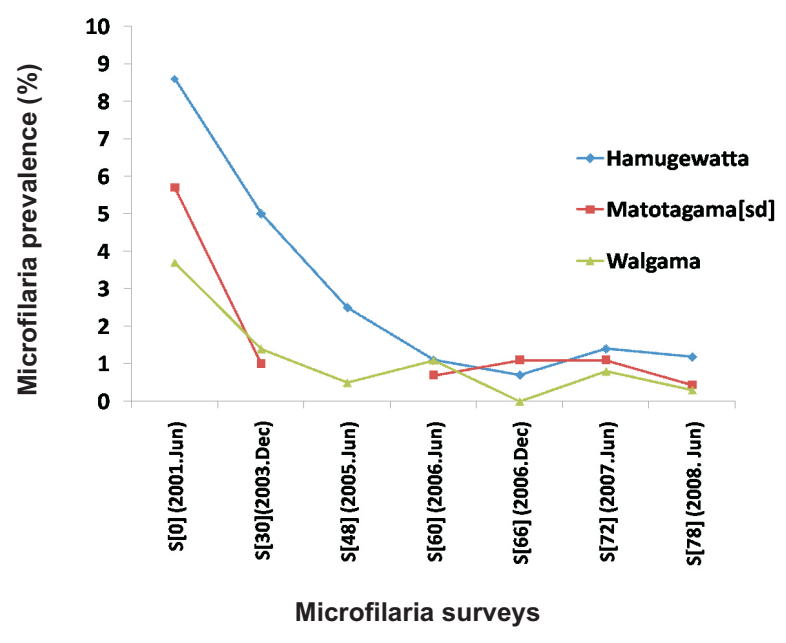

Figure 6: Changes in microfilaria (mf) prevalence following MDA's over seven years from June 2001 to June 2008
Number of mosquitoes dissected each year (August July) from resting catches of Culex quinquefasciatus in the three villages in Walgama suburb were used to generate infection rates. Fairly high infection rates were recorded before MDA. Of them Walgama had highest infection rate -5.26 in 2000/2001 collection year. It was 2.67 and 1.82 in Walgama Central and Matotagama respectively. After successful MDAs infection rate reached zero in 2007/2008 collection year and remained zero thereafter. This infection rate reduction was statistically significant in all GNs $(\mathrm{P}<0.01)$ (Figure $7 \mathrm{a})$. Very low numbers of $\mathrm{L} 3$ larvae were detected in all three villages before MDA. However, infectivity rates reached zero by 2005/2006 collection year (Figure 7b.) (19).

\section{Infection rates}

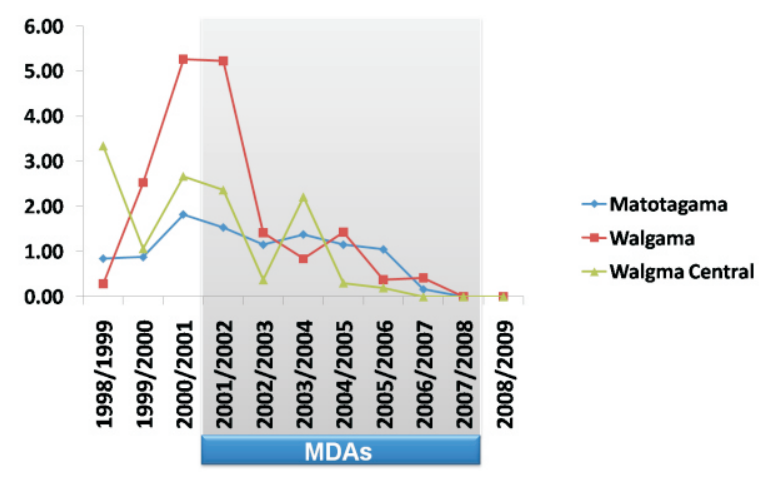

Figure 7a: Changes in mosquito infection rates IDA, MDA and post-MDA periods

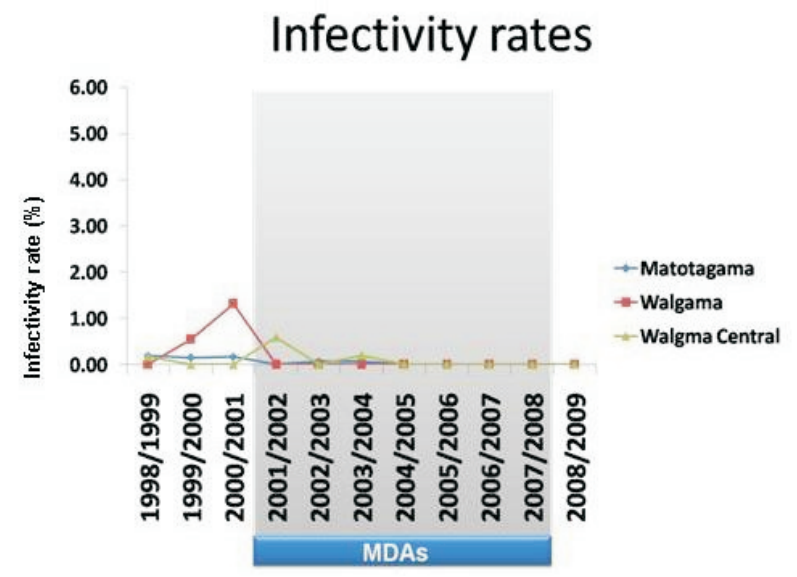

Figure 7b: Changes in mosquito infectivity rates pre-MDA, MDA and post-MDA periods 
Anti-filarial MDAs provide a cost-effective control method for STH, which was witnessed in our study areas. Following 6/12 rounds of anti-filarial MDAs cumulative pre-MDA STH prevalence (11-55\%) reached very close to zero (Figure 9) (17).

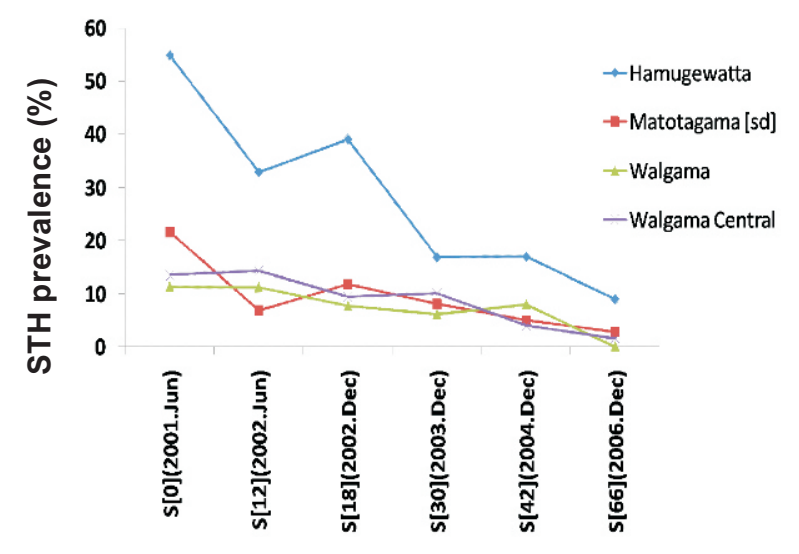

Figure 9: Changes in prevalence of Soiltransmitted helminths (STH) following MDA's over five years from June 2001 to Dec. 2006

ELISA for filaria-specific IgG4 in urine (urine ELISA) was applied to children in 7 schools in Deniyaya, Sri Lanka, before and after 5 rounds of annual mass drug administration (MDA). The pretreatment $\mathrm{IgG} 4$ prevalence in 2002 was $3.20 \%$, which decreased to $0.91 \%$ in 2003 after the first MDA $(P<0.001)$, and finally to $0.36 \%$ in 2007 after the $5_{\text {th }}$ MDA. Among 5-10 year-old children, the prevalence decreased from $3.37 \%$ in 2002 to $0.51 \%$ in $2003(P<0.01)$. A pattern of IgG4 titer distribution according to age and its yearly change could also provide useful information in drug efficacy analysis. In 2008, new samples from eleven 2006/07 urine ELISA-positive students and their family members $(n=56)$ were examined by ICT $-W$. bancrofti antigen test, microfilaria test, and urine ELISA. No infection was confirmed among them. Urine ELISA will be useful in monitoring extinction/resurgence in a postMDAlow endemic situation(19).

\section{Step 3 - Surveillance for LF, repeat TAS, and re-mapping}

National PELF had selected Sentinel Sites (Two sentinel sites per million people) and continues monitoring in endemic areas. In our surveillance programme we had selected one spot check site within the Walgama suburb where MDAs was conducted by our team and another site near Galle town where MDAs were conducted by the national PELF. The total population resident in the villages was screened with ICT and urine ELISA in August 2009 (20). In Hamugewatta, children aged 2-10 years, the ICT rate was $0.9 \%(1 / 111)$ and the urine ELISA rate $2.5 \%(3 / 119)$. IgG4 titers of the 3 positive children were low (less than $100 \mathrm{U}$ ). In Unawatuna-West, basically similar results were obtained: ICT rate of $2-10$ yrs $0 \%(0 / 69)$, ELISA rate of $2-10 \mathrm{yrs} 2.7 \%(2 / 73)$, and IgG4 titers of the 2 children $<100 \mathrm{U}$.

\section{Step 4 - Verification}

Presently we are at the end stage of step 3 and collecting evidence for verification (Figure 2). A study was carried out using same RAP to screen one borderline district (Ratnapura) (Figure 1) to demonstrate the existence of an undocumented endemic focus (21). ICT or urine ELISA or microfilaraemia was used to confirm the existing infection. According to $G N s^{\prime}$ response five $G N$ divisions with highest number of hydrocoele cases were selected [Batugedara (Hydrocoele: 1000 males = 11.7), Olugantota (7.8), Kospalavinna (5.9), Seelogama (5.5), Angammana (4.9)] and ICT or urine ELISA or microfilaraemia survey was conducted. Urine was tested for IgG4 in all residents $<25$ years in selected five $G N$ divisions. Highest urine ELISA positive rate was in Batugedara (1.64\%, CI 0.67-3.63). Urine ELISA positives and their family members in Kospalavinna and Seelogama were retested with ICT and night blood film for microfilaria. All rates were nil except ICT rate in Kospalavinna (1.8\%, CI 0.01-10.52). Rest of the $G N$ divisions is yet to be tested with ICT / $\mathrm{mf}$ surveys and selected divisions with entomology surveys.

We have developed a loop-mediated isothermal amplification (LAMP) method to detect Wuchereria bancrofti DNA (22). The sensitivity and specificity of LAMP method were equivalent to those of PCR method which detects $S s p$ I repeat sequence in $W$. bancrofti genomic DNA: both methods detected one thousandth of $W$. bancrofti DNA from one microfilaria (Mf), and did not cross-react with DNAs of Brugia malayi, B. pahangi, Dirofilaria immitis, human and Culex quinquefasciatus. 
We also examined the sensitivity of LAMP using the mimic samples of patient's blood or blood-fed mosquitoes containing one $W$. bancrofti Mf per sample. The LAMP method was able to detect $W$. bancrofti DNA in $1,000 \mu \mathrm{l}$ of blood or in a pool of 60 mosquitoes, indicating its usefulness in detecting/monitoring $W$. bancrofti infection in humans and vector mosquitoes in endemic areas.

We plan to carry out school based (targeting children 6-7yr old) ICT, urine ELISA surveys and LAMP method for entomological analysis in Matara, Galle (Known endemic areas), Batticaloa and Jaffna (Uncertain endemic areas) educational divisions according to new WHO protocol (23). The results generated by said studies will be much needed data to national PELF and WHO in their process of national and global eradication LF respectively.

\section{Disability Prevention and Control}

Disability prevention and control are the management of suffering caused by existing filarial disease.

\section{Living with LF}

In a study in Matara before the initiation of a community home based care (CHBC) programme the situation of 101 patients was examined. Subjects with severe lymphoedema (grade III or higher) were more prone (24 of $31,77.4 \%$ ) to develop Acute Inflammatory Episodes (AIEs) than the lesser grade lymphoedema (grades I and II, 34 of 58, 58.6\%). There was no indication of any active practice of limb care, and the routine hygiene of the lymphoedema cases was generally poor. Many of the cases made no attempt to wash their affected limbs between baths, and those who did wash their limbs did not do it in the best way [i.e. as recommended by Dreyer, et al. (2002)], often using too abrasive, hard and stiff material to clean their skin. Only a few cases used a clean cloth to dry their limbs (24). Majority were too busy during the day to have their limbs elevated. Only 5 cases exercised their limbs, and many (almost 43\%) never used any footwear. Many of the cases, especially women, were too embarrassed to travel to government-run clinics. Most cases seeking treatment were not given information about the more effective lymphoedema management methods, presumably because many local clinicians were unaware of the developments made in lymphoedema management in the last decade (25). General hygiene was poor with limbs neglected. 32 most disabled subjects had a mean Dermatology Life Quality Index (DLQI) score of 10.3 (range 520). Fifteen of the 32 (46.9\%) were positive for entry lesions (ELs), while only 16 of 67 $(23.9 \%)$ who had $<5$ of DLIQI score did so $(\mathrm{P}<0.05)$ (26).

Using same group of people we looked in to disease impact on their psychosocial parameters (27). The study found that LF was extremely debilitating to participants over long periods of time.

\section{Monitoring of CHBC}

In a study to monitor CHBC, our team selected two groups of 15 patients each for home based care under personal observation. In this interventional study, the efficacy of (a) a daily monitoring scheme and (b) a monthly monitoring scheme was compared after one year of care.

27 lymphoedema patients who had Grade II or more lymphoedema with or without EL, and history of AIEs were enrolled in the follow-up study. Three of them not accepted continuous visits of our team. 14 who had their homes close to one another were selected for a daily monitoring scheme (DM) and the balance 13 was followed up monthly (MM). The total number of AIEs occurred in 1 year was computed to obtain AEIs frequency and a water displacement technique was adopted to measure the volume. Identical photographs were taken to visualize any obvious change. DLQI by Finlay and Khan was used to assess the quality of life. A significant alleviation of AIEs and EL was observed after one year in both monitoring schemes. In the long term, DM was better since it provided significantly higher KAP scores on lymphoedema management protocol, benefit score, significant reduction of limb volume, and significant improvement in QoL compared to MM (28).

A qualitative assessment of each case was done following in-depth direct interviews. Three of several success stories are described below. 
Evidence of improvement in the appearance of limb and skin hygiene (29): Siriya was born in 1933. "35 years ago when I was bearing my first child I first noticed a painful reddish enlargement at right lower limb. Later it was diagnosed as elephantiasis and got regular medication for five years. Then stopped all medications because my leg was not improved with it. I took medicine only when I got fever and limb pain which I got at least 3 to 4 times per year. Now I am looked after by my grandson and his wife. My husband is also a chronic patient. My grandson's family has lots of financial and mental problems due to both of us. How can I even cope-up with day to day activities with this large leg (photograph A - taken after first washing)" This was her first dialogue with our team. Then our team trained them to carryout CHBC. A field assistant visited her home daily and facilitated her family to implement CHBC programme while identifying existing problems. All the identified problems were addressed by the senior author in his weekly house visits. After one year she had shown a remarkable improvement in her limb size (photograph B). Siriya "Oedema has reduced dramatically, now I feel lightness in the affect side, now I manage to go to toilet without others help, no fever attacks thereafter, my family is very much relieved with this miracle improvement."

Siriya's husband "Now it's very difficult keep her inside always go out and gossip with her friends." Siriya's granddaughter-in-law "No fever attacks, it is a great relief for us, earlier we had to spend 1000-1500 SLR (10-15 USD) per episode. Villagers are talking about her improvement and they are very much satisfied about new care over the conventional methods."

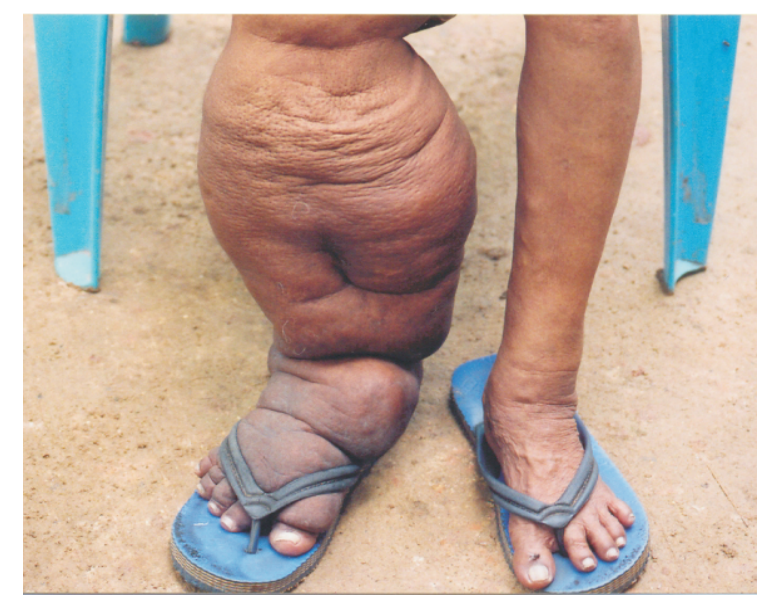

(A)

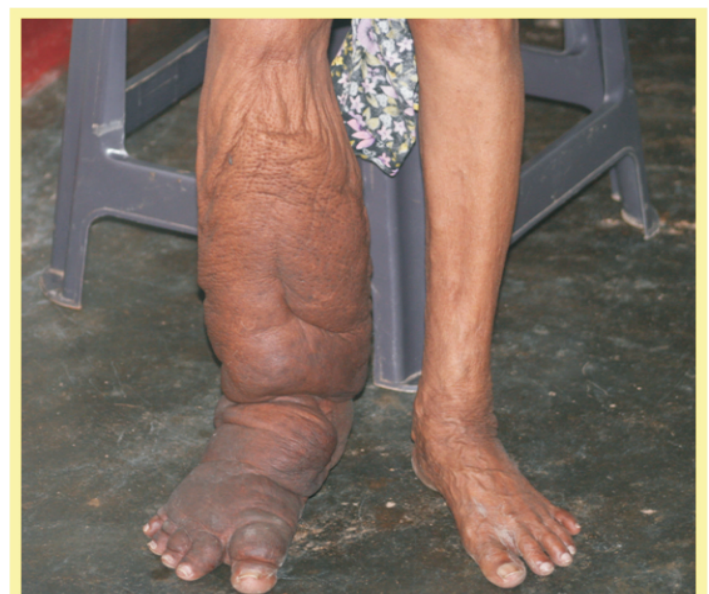

(B)

Evidence of changes in the lymphoedema grade (29): Mala was looked after by her son. She first experienced her painless pitting oedema when she was 30 years old.

Past 18 years or so she had many troubles due to this oedema. "I got severe cellulitis attacks even after a small scratch over the affected limb. I had to experience two/ three such attacks per year in the past. Sometimes I was addmitted to the government hospital. My son has to bare all the expenses.

My husband is home bounded due to a nerve problem, he can not work anymore.

Most of the time I was treated by a General Practitioner who conducted a private clinic in our village. Medication resolved my acute problems but I got these fever attacks regularly. I do all family works at home, hardly any time to look after my limb. Inever wore a pair of slippers even when I engaged work in the home garden." Her son and she were given an adequate knowledge regarding CHBC. A volunteer visited her daily. Senior author visited her weekly. CHBC was adapted to suit to her life style. Mala "This is the first time a medical personnel visited our house and talked to us.

There is slight reduction in oedema. With the girl who visited me every day I washed my limb but other things, I mean limb elevation and exercises were not done properly. During day time I couldn't get a chance to elevate limb but I managed to keep it elevated during sleep. I had a skin creases over here (red circles photograph C) but now it has disappeared (photograph D). I feel that the skin is also smoother than previous. I didn't get a single attack of fever for the last year, it is a great thing otherwise my poor son has to suffer a lot." Mala's 
husband "No fever attacks like those days, it is a great relief. She couldn't do the whole programme because she has to look after both me and son. However, a reduction of oedema is seen".

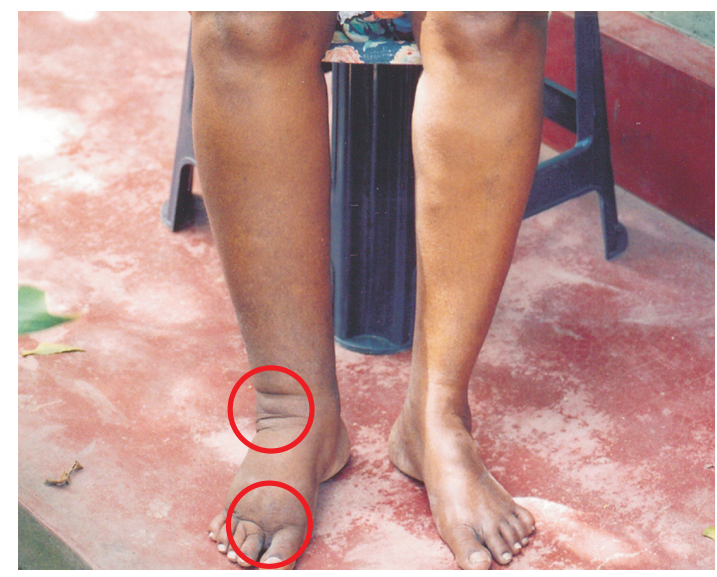

(C)

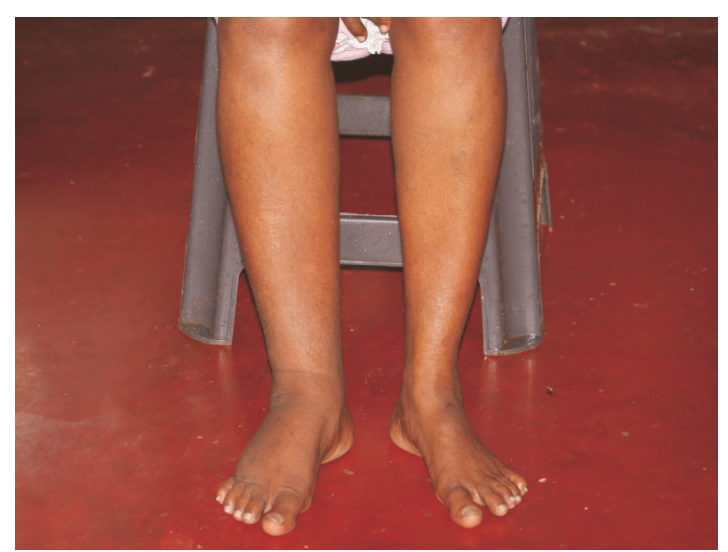

(D)

Evidence of improvement of Quality of Life (QOL) (29): Nanda was 55 years old when she first met us. She is an unmarried woman who lives with her mother (78yrs). Mother has to look after Nanda despite of her age. She was home bounded and just sit on a chair for the whole day.

To her knowledge she beared this limb oedema for more than 45 years: "I took medicine for a small oedema for about six years from government hospital, they gave me monthly injections instead of oral penicillin tablets. I stopped going there because my mother could not afford travelling. I also didn't want to go out because I want to hide my limb from villagers. After stopping regular injection I developed many fever attacks, sometimes I had to hospitalize for several days.

With time oedema got worsen and lots of skin nodules and ulcers were appeared. Very recently I had a fall due to imbalance of the limb and got a fractured leg bone." Nanda and her mother was introduced to the new limb management protocol (CHBC).

She couldn't do elevation and exercises properly at the beginning of the programme due to the fractured bone. Our volunteer visited her daily and helps her and her mother to implement the CHBC protocol. Senior author has recommended her to carry out series of physiotherapy: "When I started regular washing the bad odour of the limbs disappeared and it was a great relief. Oedema too got reduced to certain extent. I would have achieve all these improvement well ahead unless I had broken leg. Now I can walk alone. Now I go to the temple with my mother on Poya days. No fever attacks has saved significant amount of money to us. Earlier days I had small nodules, warty like lesions over the affected limb and many skin infections specially in between two webs (photograph E). I am very fortunate, now I can't see those lesions" (photograph F). Nanda's mother: "Now I can take her to a public place because she doesn't have bad limb smell like those days. There are no fever attacks, it a big relief for me."

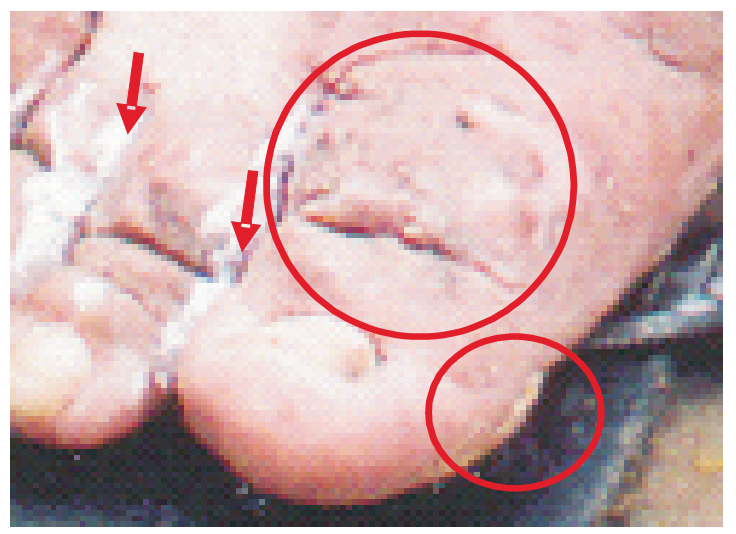

(E)

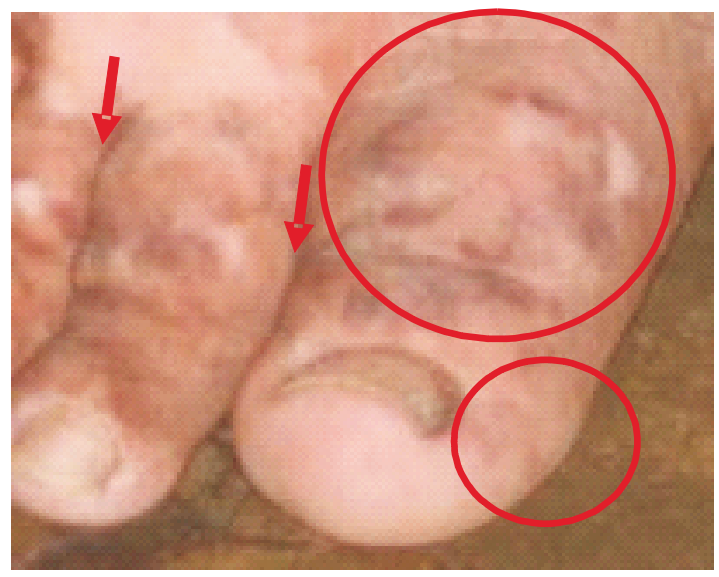

(F) 


\section{Summery and Conclusions}

The estimate of community hydrocoele prevalence obtained by ordinary people (IndQ) showed a positive and significant correlation with the clinical levels determined by clinicians (CE) and the prevalence levels by urine ELISA. Therefore, ordinary people's information on lymphatic filariasis prevalence can be used as preliminary information to identify endemic areas. Further, results suggested that IndQ worked reasonably well even in a low endemic area. Therefore, the same IndQ approach can be applied in the districts where the MDA programme had not been done, but the presence of hidden endemic foci cannot be ruled out. The study benefited much from the existence of an efficient and reliable mailing system in the country. However, similar indirect method based on questionnaire would be applicable in other countries where there is not enough health manpower but with the understanding and support of local people.

Development of digitized maps of filariasis endemicity in Southern Sri Lanka using GIS technology, has provided several new insights relevant to national PELF. The maps draw attention to the substantial burden of the LF and close approximation of true spatial extent of the problem within the three districts of the Southern Province of the country. In Galle, the information by 690 local leaders revealed clearly an aggregated distribution of elephantiasis/lymphoedema in the $10 \mathrm{~km}$-wide coastal zone, which mostly overlaps with the longstanding most endemic "filaria belt" described by Sasa (3). In contrary, hydrocoele was found widespread in the district. One possible explanation for different distribution could be that the history of endemic filariasis in most inland areas is not longer than 40 years, which would not be enough for elephantiasis to make appearance. However, further investigations are needed to clarify if hydrocoele can be produced in a low transmitting area.

We have observed that some of the high prevalent foci were found near the borders with non-endemic inland districts. This fact would suggest a possible existence of endemic foci within the adjoining nonendemic districts like Ratnapura and Kegalle. Such foci could have been missed leading to a premature stoppage of MDA. Therefore, the national PELF should adopt the RAP to screen neighbouring districts and apply elimination measures before final declaration of LF elimination. If the presence of endemic foci is indicated, confirmation could be done using direct tests like ICT or ELISA. This would eventually cut down the expenses and time in the elimination programme.

An intensive IEC programme prior to each MDA and house-to-house drug delivery by a team that included a medical doctor achieved a near $100 \%$ compliance. Doctors who worked as a member of house visiting team played a critical role. They were able to convince the people who had doubts about MDA. Drug delivery by a doctor is not practicable anywhere, but well organized propaganda programme with the cooperation of health personnel such as medical students, pupil nurses, public health students, and village leaders $(G N \mathrm{~s})$ as volunteers could improve drug delivery and compliance.

Significantly lower coverage observed in municipalities, specially in Colombo Municipality. In depth analysis of coverage in Colombo, showed that the low percentages were mainly due to non participation of upper class houses. Generally, LF prevalence is lower among such upper class families than that of the poor. Therefore, we believe that the impact created by such families on coverage may not jeopardize the national PELF. However, close monitoring should be continued including upper class households in the verification stage.

We conducted an intensive study to assess the impact of the single dose multi-round MDA by carrying out mf surveys prior to each drug delivery. Our study showed that a community wide annual/biannual treatment with a single dose could suppress $\mathrm{mf}$ prevalence and density to a significantly low level. We confirm the use of single dose multi-round MDAs as a strategic tool to interrupt transmission.

GPELF had set a criterion, based on the Chinese experience, that residual filarial infections disappeared without further intervention after chemotherapy achieved $<1 \% \mathrm{mf}$ prevalence by night blood thick smear. This scenario could be expected in many endemic areas of the country. However, it is noteworthy that even after completion of 13 MDAs, in one of our study divisions which had relatively higher pre-MDA mf prevalence did not reach the targeted $\mathrm{mf}$ level of $<1 \%$. Such endemic foci could exist in other parts of the endemic zone of the country. The division under review had $>1 \% \mathrm{mf}$ rates despite near total coverage rates would imply the 
presence of some people who did not respond to treatment. In fact, 4 of $7 \mathrm{mf}$ positives encountered in the final survey had been positive on more than 3 previous occasions. They could be a future source of infection and may need special attention in the postMDA monitoring.

Vector infection/infectivity rates play a major role in monitoring and confirming interruption of transmission. In our area both rates have been declined significantly after 7-13 rounds of MDA. However, the crude dissection of mosquitoes collected by resting catching remained negative despite of having $\mathrm{mf}$ positive cases in the same cluster. This may be due to low sensitivity of crude dissection method. Therefore our team tested a more sensitive method, LAMP to find out mosquito infections by assessing parasite's DNA successfully.

Apart from tests for $\mathrm{mf}$, circulating filarial antigen (CFA) and anti-filarial antibody has been used in our studies specially covering young children who were born after the commencement of the MDA to detect any change in transmission. The five year follow up study carried out in Deniyaya showed that urine ELISA will be useful in monitoring extinction/ resurgence in a post-MDA low endemic situation. Further results suggested that the level of filarial transmission is now very low, and this was confirmed by a more sensitive CFA test among young children.

Following 6/12 rounds of anti-filarial MDAs, $A$. lumbricoides and hookworm infections reached zero prevalence and T. trichiura infection was suppressed to a very low level. This significant decline in the Soil Transmitted Helminth (STH) prevalence must be attributed to MDAs which included albendazole. Anti-filarial MDAs provided a cost-effective control method for STH which was witnessed in our study divisions.

In the lymphoedema study, we observed that the subjects with severe lymphoedema (grade III or higher) were more prone to develop acute inflammatory episodes (AIE) than the lesser grades. Dermatology Life Quality Index (DLQI) has been accepted in the U.K. and applied to many skin conditions to measure patients' quality of life (QoL). The higher the total score is the more impaired is the QoL. In the present study, we observed that mean DLQI score was 10.3 (range 520) among the 32 most disabled subjects. DLQI can be adopted to measure the QoL of lymphoedema cases in Sri Lanka.
Hardly any research has been done to assess the extent and intensity of education required for patients to become competent in lymphoedema selfcare. Present study showed that the daily reinforcement of the lymphoedema management protocol had an added advantage over a monthly reinforcement. Photographs with obvious improvements in limb size and skin appearance would be valuable for propaganda and education. Irrespective of monitoring schemes, in all cases a significant alleviation of AIEs and EL was observed after one year. That invariably would have been led to a significant improvement in QoL among them. This suggests that much success could be achieved in the future by subjecting the lymphoedema cases to CHBC programme.

\section{Acknowledgements}

Authors would like to acknowledge Professors E. Kimura, M. Itoh and Y. Isogai and Doctors H Takagi, F Nagaoka for their kind acceptance for using raw data of our collaborative publications in the preparation of this article. Financial assistance was received from three agencies, University of Ruhuna Research Grant, Lymphatic Filariasis Support Centre, Liverpool School of Tropical Medicine, UK and Japanese Government Grants. Prof. N.K. Gunawardena, Dr. Rohan Dharmadasa and Dr. Danujaya Mahesh are acknowledged for their kind assistance in clinical surveys. Staff attached to FRTSU, Mr. Saman Weerasekara, K. Vidanapathirana, Achala Sampath, Theekshana Liyanage, Nihal Jayawardana and Manoj Priyanga were helpful in the field as well as in the laboratory.

\section{References}

1. Gautamadasa CH. A historical review of Brugian filariasis and its present status in Sri Lanka. M.D.Colombo: University of Colombo, 1986.

2. Anon. Filariasis: a manual for health personnel. Colombo: Anti-Filariasis Campaign, Ministry of Health and Indigenous Medicine. 1999: pp.27-38.

3. Sasa M. Human Filariasis. A Global Survey of Epidemiology and Control Tokyo: University Park Press. 1976: pp.372-7.

4. Anon. Annual Health Bulletin. Colombo: Ministry of Health and Indigenous Medicine, 2003: pp.56-8. 
5. World Health Organization (2000). Preparing and implementing a national plan to eliminate lymphatic filariasis. Geneva: WHO, WHO/CDS/CPE/CEE/2000.15.

6. World Health Organization (1998). Rapid assessment procedure for lymphatic filariasis: report on a multicountry study. Geneva: WHO, WHO/TDR/TDF/ComDT/ 98.1.

7. Weerasooriya MV, Isogai $\mathrm{Y}$, Itoh $\mathrm{M}$, Yahathugoda TC, Vidanapathirana KK, Mudalige MP and Kimura E. Distribution of filarial elephantiasis and hydrocoele in Matara district, Sri Lanka, as reported by local leaders, and an immunological survey in areas with relatively high clinical rates. Parasitology International 2008; 57: 390-5.

8. Yahathugoda TC, Weerasooriya MV, Itoh M. and Kimura E (2009). Identification of endemic areas of bancroftian filariasis in Hambantota district, Sri Lanka, using Rapid Assessment Procedures (RAPs). $10^{\text {th }}$ International Symposium on Vectors and Vector Borne Diseases, November 2009, Goa, India. (P103), pp.171.

9. Yahathugoda TC, Weerasooriya MV and Kimura E (2010). A Rapid Assessment Procedure (RAP) to assess distribution of Lymphatic Filariasis using Geographical Information Systems (GIS). The $12^{\text {th }}$ International Congress of Parasitology, August 2010, Melbourne, Australia. (abs\#1945). Available at: http://www.icopaxii.org/images/stories/final\%20web\%20 program.htm [Accessed 28 September 2010].

10. World Health Organization (1999). Report of a WHO informal consultation on epidemiologic approaches to lymphatic filariasis elimination: initial assessment, monitoring, and certification. Geneva: WHO, WHO/FIL/99.195.

11. Weerasooriya MV, Weerasooriya TR, Gunawardena NK, Samarawickrema WA \& Kimura E. Epidemiology of bancroftian filariasis in three suburban areas of Matara, Sri Lanka. Annals of Tropical Medicine and Parasitology 2001; 95(3): pp.263-73.

12. Yahathugoda TC, Wickramasinghe D, Liyanage TS, Weerasooriya MV, Mudalige MPS, Waidyaratne EI, Samarawickrema WA. Knowledge on Lymphatic Filariasis and the response to July 2002 Mass Treatment Campaign of two communities in the Galle District. Ceylon Medical Journal 2003; 48: 74-7.

13. Weerasooriya MV, Yahathugoda TC, Wickramasinghe D, Gunawardena NK, Dharmadasa RA, Vidanapathirana KK, Weerasekara SH, Samarawickrema WA.. Social mobilisation, drug coverage and compliance and adverse reactions in a Mass Drug Administration (MDA)
Programme for the Elimination of Lymphatic Filariasis in Sri Lanka. Filaria Journal 2007; 6: 11.

14. Weerasooriya MV, Yahathugoda TC, Samarawickrema WA. 2006. Coverage and compliance during 2004 Mass Drug Administration of the Programme for the Elimination of Lymphatic Filariasis in the Municipality of Colombo, Sri Lanka. $11^{\text {th }}$ International Congress of Parasitology, August 2006, SECC, Glasgow, Scotland. File://G: ICOPA06\index.html.

15. Weerasooriya MV, Yahathugoda TC, Samarawickrema WA (2006). Impact of an effective propaganda strategy prior to the 2004 Mass Drug Administration (MDA) on drug delivery in the Galle Municipality, Sri Lanka. $11^{\text {th }}$ International Congress of Parasitology, August 2006, SECC, Glasgow, Scotland. File://G:IICOPA06\index.html.

16. Yahathugoda TC, Weerasooriya MV, Kimura E (2009). Annual Mass Drug Administration in Walgama, a suburb of Martara, Sri Lanka and its evaluation. Xth International Symposium on Vectors and Vector Borne Diseases, November 2009, Goa, India. (P104), pp.172.

17. Yahathugoda TC, Weerasooriya MV and Kimura E (2010). Effects of repeated rounds of anti-filarial Mass Drug Administration (MDA) with diethylcarbamazine (DEC) plus albendazole on Soil Transmitted Helminths (STHs) in Sri Lanka. The $12^{\text {th }}$ International Congress of Parasitology, August 2010, Melbourne, Australia.(abs\#1944). Available at: http://www.icopaxii.org/images/stories/final\%20web\% 20program.htm [Accessed 28 September 2010].

18. Yahathugoda TC, Weerasooriya MV, Mudalige MPS, Samarawickrema WA. The impact of single-dose mass drug administration of diethylcarbamazine combined with albendazole given annually and biannually over six years on the transmission of Wuchereria bancrofti by Culex quinquefasciatus in Walgama, Matara. Galle Medical Journal 2011; 16(2): 46-47.

19. Itoh M., Weerasooriya MV, Yahathugoda TC, Takagi H, Samarawickrema WA, Kimura E. Effects of 5 rounds of Mass Drug Administration with diethylcarbamazine and albendazole on filaria-specific IgG4 titers in urine: 6-year follow-up study in Sri Lanka. Parasitology International 2011; 60,393-7.

20. Weerasooriya MV, Yahathugoda TC, Itoh M, Kimura E (2010). Urine ELISA as a tool for the confirmation of filariasis elimination or the early detection of resurgence: a study in Galle and Matara districts, Sri Lanka. Joint International Tropical Medicine Meeting 2010 and International Malaria Colloquium 2010. (poster\#49). Availableat: http://www.jitmm.com/uploadDoc/ 12902256030037.pdf[Accessed 14 January 2011]. 
21. Yahathugoda TC, Weerasooriya MV, Takagi H, Itoh M \& Kimura E. National Programme for the Elimination of Lymphatic Filariasis in Sri Lanka: A study to confirm the non existence of endemic foci of bancroftian filariasis in a district not covered by mass drug administration. Galle Medical Journal 2011; 16(2): 47-48.

22. Takagi H, Itoh M, Kasai S, Yahathugoda TC, Weerasooriya MV, Kimura E. Development of a loop mediated isothermal amplification method for detecting Wuchereria bancrofti DNA. Parasitology International 2011; 60: 493-7.

23. World Health Organization (2011). Global Programme to Eliminate Lymphatic Filariasis: A manual for National Elimination Programmes. Geneva: WHO.

24. Dreyer G, Addiss D, Dreyer P, Noroes J. 2002. Basic Lymphoedema Management. Hollis, NH: Hollis Publishing Company.

25. Subasinghe GP, Yahathugoda TC, Wickramasinghe D, Gunawardena NK, Dhanuja Mahesh KH, Weerasooriya MV, Samarawickrema WA. Awareness of the National Programme for Elimination of Lymphatic Filariasis (PELF) among doctors doing private practice. Sri Lanka Medical Association $119^{\text {th }}$ Anniversary Academic Sessions, 2006; 51: 42 .
26. Yahathugoda TC, Wickramasinghe D, Weerasooriya MV, Samarawickrema WA. Lymphoedema and its management in cases of lymphatic filariasis: the current situation in three suburbs of Matara, Sri Lanka, before the introduction of a morbidity-control programme. Annals of Tropical Medicine and Parasitology 2005; 99: 501-10.

27. Perera M, Whitehead M, Molyneux DH, Weerasooriya MV, Gunatilleke G (2007). Neglected patients with a neglected disease? A qualitative study of lymphatic filariasis. PLoS Neglected Tropical Diseases, [Online]. 21 Nov., 1, e128. Available at: http://www.plosntds.org/article/info\% 3Adoi\%2F10.1371\%2Fjournal.pntd.0000128 [Accessed 3 August 2008].

28. Yahathugoda TC, Weerasooriya MV, Kimura E (2010). Evaluation of two monitoring schemes after one year of Community Home-Based Care (CHBC) programme of morbidity control in Lymphatic Filariasis in three suburbs of Martara, Sri Lanka. The $12^{\text {th }}$ International Congress of Parasitology, August 2010, Melbourne, Australia. (abs\#157). Available at: http://www.icopaxii.org/images/ stories/final\%20web\%20program.htm [Accessed 28 September 2010]. 